\title{
Does lavender aromatherapy alleviate premenstrual emotional symptoms?: a randomized crossover trial
}

\author{
Tamaki Matsumoto $^{1 *}$, Hiroyuki Asakura ${ }^{2}$ and Tatsuya Hayashi ${ }^{3}$
}

\begin{abstract}
Background: A majority of reproductive-age women experience a constellation of various symptoms in the premenstrual phase, commonly known as premenstrual syndrome (PMS). Despite its prevalence, however, no single treatment is universally recognized as effective, and many women turn to alternative approaches, including aromatherapy, a holistic mind and body treatment. The present study investigated the soothing effects of aromatherapy on premenstrual symptoms using lavender (Lavandula angustifolia), a relaxing essential oil, from the perspective of autonomic nervous system function.

Methods: Seventeen women ( $20.6 \pm 0.2$ years) with mild to moderate subjective premenstrual symptoms participated in a randomized crossover study. Subjects were examined on two separate occasions (aroma and control trials) in the late-luteal phases. Two kinds of aromatic stimulation (lavender and water as a control) were used. This experiment measured heart rate variability (HRV) reflecting autonomic nerve activity and the Profile of Mood States (POMS) as a psychological index before and after the aromatic stimulation.

Results: Only a 10-min inhalation of the lavender scent significantly increased the high frequency (HF) power reflecting parasympathetic nervous system activity in comparison with water (aroma effect: $F=4.50, p=0.050$; time effect: $F=5.59, p=0.017$; aroma $x$ time effect: $F=3.17, p=0.047)$. The rate of increase in HF power was greater at 10-15 $\min (p=0.051)$ and $20-25 \mathrm{~min}(p=0.023)$ in the lavender trial than in the control trial with water. In addition, POMS tests revealed that inhalation of the aromatic lavender oil significantly decreased two POMS subscalesdepression-dejection $(p=0.045)$ and confusion $(p=0.049)$ —common premenstrual symptoms, in the late-luteal phase, as long as 35 min after the aroma stimulation.

Conclusions: The present study indicated that lavender aromatherapy as a potential therapeutic modality could alleviate premenstrual emotional symptoms, which, at least in part, is attributable to the improvement of parasympathetic nervous system activity. This study further implies that HRV could evaluate the efficacy of aromatherapy using various fragrances to relieve premenstrual symptoms, and ultimately, support the mind and body health of women.
\end{abstract}

\section{Background}

A majority of women experience at least some degree of a regular recurrence of various biopsychosocial symptoms during the days prior to menstruation, which usually abate following menstruation. The cluster of symptoms can alter behavior and well-being, and affect family, friends, and working relationships. We commonly know this enigmatic

\footnotetext{
* Correspondence: tamaki@shitennoji.ac.jp

1 Department of Health Education, Faculty of Education, Shitennoji University,

3-2-1 Gakuenmae, Habikino Osaka 583-8501, Japan

Full list of author information is available at the end of the article
}

condition appears in the late-luteal phase as premenstrual syndrome (PMS) [1-3]. Problematic symptoms of PMS may begin in the adolescent years, although the most severe symptoms occur in the 20s to mid-30s [1]. Symptoms and discomfort levels of PMS vary from woman to woman. Researchers have attempted to unveil this unique condition from various perspectives. At the time of writing, however, no consensus had emerged on which specific physical findings, biological markers, or laboratory tests comprise a diagnosis and/or etiopathogenesis of PMS [1-3]. Apart from complete elimination of the menstrual

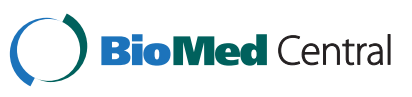


cycle, no single treatment is universally recognized as effective, and many women often turn to therapeutic approaches outside of conventional medicine [4].

The practice of aromatherapy - $\mathrm{a}$ form of complementary and alternative medicine-uses volatile plant materials, known as essential oils, and, with its ancient roots, has a long history of use in supporting women's health and lifestyles $[5,6]$. While some predominantly anecdotal therapeutic evidence derives from small trials and case studies, aromatherapy has gained a resurgence of interest as women seek safe and efficacious options for their healthcare. Lavender, a representative of relaxing essential oils, enjoys popularity and wide use in the clinical fields of psychosomatic obstetrics and gynecology. Therapeutic treatments with lavender oil help women cope with labor pain [6], ease post-cesarean pain [7], reduce postpartum depression and anxiety [8], decrease dysmenorrhea [9], and alleviate climacteric symptoms including melancholia, hot flushes, arthralgia, and myalgia [10]. A short term of inhalation of essential oils containing linalool and linalyl acetate-two major components of lavender-also improves mood states during pregnancy [11] and insomnia among midlife women [12]. Taking these findings into consideration, we find it plausible that aromatherapy with lavender fragrance could ameliorate PMS. A literature search for this study using the PubMed database, a service of the US National Library of Medicine and the National Institutes of Health, however, identified no available empirical research regarding the efficacy of lavender on PMS.

Although all body systems contribute, the relative stability of the human internal environment depends largely on the orchestrations of the autonomic nervous system, comprising two divisions-sympathetic and parasympathetic nervous systems. Instability, or even a slight disorder of the autonomic nervous system, therefore, could induce broadly ranging psychophysiological phenomena. Biofluctuomatics technology allows us to explore the functioning of the autonomic nervous system reliably and noninvasively using comprehensive and functional analysis of heart rate variability (HRV). Researchers have consistently observed reduced HRV during and following acute and chronic stress $[13,14]$. Men and women exhibiting depression [15], anxiety [16], post-traumatic stress disorder [17], and chronic fatigue syndrome [18] also experience diminished HRV. Thayer et al. [19], in addition, found emotion regulation linked to HRV, i.e., individuals with greater emotion-regulation ability have been shown to have greater levels of resting HRV. Studies have investigated psycho-physiological effects of aromatherapy by using HRV and demonstrated significant changes of autonomic nervous system activity after inhalation of essential oils, including lavender, while affecting mood, mental states and behavior [20]. These findings demonstrate the functionality of HRV measurements to evaluate effects of aromatherapy on premenstrual symptomatology from the perspective of autonomic nervous system activity as it reflects mind and body interaction.

Accordingly, the present study designed a randomized crossover study to evaluate the efficacy of commercially available lavender oil on mood states and autonomic nervous system activity by using HRV measurements in the late-luteal phase of eumenorrheic women. We then investigated whether inhalation of lavender fragrance could serve as an alternative method to alleviate undefined psychoemotional conditions most reproductive-age women experience premenstrually.

\section{Methods}

\section{Subjects}

Seventeen women in their 20s volunteered to participate in this study. The women, all college students, responded to a campus advertisement. The study protocol was approved in advance by the Institutional Review Board of Shitennoji University and was performed in accordance with the Declaration of Helsinki of the World Medical Association. All subjects received an explanation of the nature and purpose of the study: to investigate soothing effects of plant fragrance on emotional symptoms in the premenstrual phase. We did not, however, inform subjects of which fragrance we would use for the experiments. Prior to receiving any data about the experiments, all subjects gave their written informed consent to participate in the study.

The subjects underwent medical examinations and interviews and completed a standardized health questionnaire regarding medical history, medications, current health condition, regularity of menstrual cycle, premenstrual discomfort, and lifestyle. While referring to subjects' self-reported regular menstrual cycles, we determined the cycle phase during the experiments by the onset of menstruation and oral temperature verified by concentrations of ovarian hormones, estrone (E1), and pregnanediol-3-glucuronide (PdG), in a urine sample taken early in the morning. Both E1 and PdG were indexed to creatinine $(\mathrm{Cr})$ excretion [21-24].

As to premenstrual discomfort, responses of participants to the health questionnaire indicated that all women had some subjective psychophysiological complaints of varying degrees, from mild to moderate. However, none of the women reported that premenstrual symptoms markedly interfered with work, school, usual activities or relationships with others. To determine the severity of premenstrual symptoms, we asked subjects to record a daily symptom diary based on the menstrual distress questionnaire [25] for at least two menstrual cycles. The average value of the increase on the total scores of the diary from the follicular (day 5 to day 11 from the first day of menstruation) to the late-luteal 
phase (within seven days before the next menstruation) was $10.3 \pm 2.4 \%$. None of the subjects reported greater than a $30 \%$ increase, a standard for diagnosing PMS $[3,26]$. Notably, according to a series of studies by the authors of this paper [21-24], a less than $20 \%$ increase of subjective symptoms from the follicular to the late-luteal phase did not influence autonomic nervous system activity during the menstrual cycle. Considering this information, no subjects in the present study suffered from severe PMS or premenstrual dysphoric disorder (PMDD), but the subjects' symptoms did fall within the sphere of premenstrual molimina (subclinical levels of premenstrual symptomatology), signaling impending normal menstruation, which a majority of reproductive-age women experience $[3,26]$.

None of the subjects had been clinically diagnosed with diabetes mellitus, hypertension, hyperlipidemia, or cardiovascular or any other endocrine or systemic disorders that could affect the autonomic nervous system. The subjects were non-obese and non-smokers. None of the women reported taking oral contraceptives to control the menstrual cycle. We did not perform a pregnancy test for this study; however, regular menstrual cycles subsequently resumed in all subjects after the completion of the study. Thus, results showed that none of the subjects were pregnant during the study period.

Referring to a study of Kiecolt-Glaser et al. [27], we performed the olfactory function test on all subjects to assure that none had anosmia. Briefly, subjects were given two sets of three bottles-two held distilled water; the third contained essential oils (lavender or orange)and were asked to choose the one that differed from the other two. To be eligible for the study, subjects had to choose the correct response in both trials.

All subjects were asked not to consume any food or beverages containing alcohol or caffeine after 21:00 of the day preceding the experiment. The subjects were also instructed to abstain from alcohol use and excessive physical activity for 24 hours before testing [21].

\section{Experimental procedure}

All subjects were examined on two separate occasions (aroma and control trials) in the late-luteal phase (within seven days before the next menstruation). The order of testing was randomized so that equal numbers of subjects were studied first in each trial. All measurements were taken between 11:00 and 15:00 and were performed in a temperature-controlled $\left(25^{\circ} \mathrm{C}\right)$, quiet, comfortable room with a minimization of arousal stimuli. Height and body weight of each subject were measured to calculate body mass index (BMI) as body weight divided by height squared. Subjects then rested for at least 10 minutes before the start of the experiment.

This experiment used two kinds of aroma stimulation: lavender (Lavandula angustifolia, Lot No. BLAH10,
Kensoigakusha Co. Tokyo, Japan) and water as a control. Major components of the lavender oil used in this study comprised linalyl acetate (37.18\%), linalool (36.83\%), trans- $\beta$-ocimene $(4.25 \%), \beta$-caryophyllene $(3.55 \%)$, cis- $\beta$ ocimene $(2.88 \%)$, and lavendulyl acetate $(2.06 \%)$. Referring to previous studies [28,29], we pipetted $10 \mu \mathrm{l}$ of lavender essential oil or water onto a small cotton pad designed for a diffuser (Aroma breeze NOVA T, ALTA Corporation, Nagoya, Japan). Airflow from the diffuser was set at $1.3 \mathrm{~m}$ per min and placed near the subject's nostrils using the diffuser's $30 \mathrm{~cm}$ long circular cylinder fitted with a perforated funnel (diameter $5 \mathrm{~cm}$ ).

Before measurements were taken, the subjects were instructed to relax quietly and comfortably for at least $10 \mathrm{~min}$ in a seated position while equipped with electrocardiograph (ECG) electrodes. They then filled out the Profile of Mood States (POMS) explained in detail below. The ECG was recorded $5 \mathrm{~min}$ before inhalation of the scent. Each subject inhaled the scent for $10 \mathrm{~min}$. We then measured the ECG for $5 \mathrm{~min}$ at $0,10,20$, and 30 min after inhalation. During ECG recording, all subjects breathed in synchrony to a metronome at 15 beats per minute to ensure that the respiratory-linked variations in heart rate did not overlap with low-frequency heart-rate fluctuations (below $0.15 \mathrm{~Hz}$ ) from other sources [21,23]. After the ECG was recorded, the subjects repeated the POMS test. The ECG signals were later analyzed by means of HRV power spectral analysis, as described below, to evaluate whether aroma stimulation changed autonomic nervous system activity.

\section{R-R interval power spectral analysis procedure}

The autonomic nervous system activity was noninvasively measured by HRV power spectral analysis, which decomposes the series of sequential R-R intervals into a sum of sinusoidal functions of different amplitudes and frequencies by the Fourier transform algorithm. The technique of the analysis for the present investigation has been applied in basic physiological and clinical research fields, and its validity and reliability has been previously confirmed [21,23,30-32]. Researchers have elsewhere described the procedure of R-R interval power spectral analysis used in the present study in great detail [30,31]. Briefly, the ECG signal was amplified (MEG-6108, Nihon Kohden, Tokyo, Japan) and digitized via a 16-bit analog-todigital converter (Model PS-2032GP, TEAC, Tokyo, Japan) at a sampling rate of $1000 \mathrm{~Hz}$. The digitized ECG signal was differentiated, and the resultant QRS spikes and the intervals of the impulses (R-R intervals) were stored sequentially on a hard disk for later analyses.

Before the R-R spectral analysis was performed, the stored R-R interval data were displayed and aligned sequentially to obtain equally spaced samples with an effective sampling frequency of $2 \mathrm{~Hz}$ and displayed on a 
computer screen for visual inspection. Then, the direct current component and linear trend were completely eliminated by digital filtering for the band-pass between 0.03 and $0.5 \mathrm{~Hz}$. The root mean square value of the R-R interval was calculated as representing the average amplitude. After passing through the Hamming window, power spectral analysis by means of a fast Fourier transform was performed on a consecutive 256-sec time series of R-R interval data obtained during the test. Spectral powers were calculated for the following respective frequency band: low frequency (LF) power $(0.03-0.15 \mathrm{~Hz}$ ), an indicator of both sympathetic and parasympathetic nervous system activity; high frequency (HF) power $(0.15-0.5 \mathrm{~Hz})$, which solely reflects parasympathetic nerve activity; and Total power $(0.03-0.5 \mathrm{~Hz})$ representing overall autonomic nervous system activity.

Basal heart rates and autonomic nervous system activities differ from individual to individual. Thus, the mean values for heart rates before inhalation of scent were set as the baseline values and the mean values for autonomic nervous system activity before the inhalation were standardized to $100 \%$. The rate of change after the inhalation was compared between aroma and control trials [28].

\section{Assessment of emotional symptoms}

We administered the Japanese version of the POMS test (Kaneko Shobo Co., Tokyo, Japan), a globally standardized, self-administered, 65-item questionnaire (including 7 dummy items) to assess premenstrual mood states before and after inhalation of the lavender aroma and water. Each item was rated on a five-point Likert-type scale of zero to four, ranging from "not at all" to "extremely." We added these raw scores to generate six subscales of emotional state: tension-anxiety, depression-dejection, anger-hostility, vigor, fatigue, and confusion. These added raw scores were then converted into $\mathrm{T}$-scores according to the POMS manual [33]. We should mention that, according to our recent study [24], five negative POMS variables-tension-anxiety, depression-dejection, angerhostility, fatigue, and confusion-reflect the cluster of premenstrual psychoemotional symptoms. In addition, those variables significantly and positively correlated in the lateluteal phase. Referring to a study by Kuroda et al. [28], to investigate the effect of lavender aroma on mood states, we compared changes in the POMS scores of the lavender and control trials before and after the ECG measurements.

\section{Statistical analysis}

To investigate the acute influence of inhalation of the lavender aroma on HRV spectral power, the effects of aroma and time and their interaction (aroma $\mathrm{x}$ time) were evaluated using two-way ANOVA with repeated measures. When significant interactions were found, we conducted paired $t$ tests between lavender and control trials and one-way ANOVA with repeated measures during each trial. When Mauchly's test of sphericity showed significance, probability values were adjusted using the Huynh-Feldt correction. Paired t tests were performed to compare changes in scores of the POMS test before and after the ECG measurements between aroma and control trials. Values are reported as mean \pm standard errors (SE). $P$ values $<0.05$ were considered statistically significant. All statistical analysis was performed using a commercial software package (IBM SPSS Statistics Version 20).

\section{Results}

\section{Clinical characteristics of subjects}

Mean values of physical features of all subjects were as follows: age $20.6 \pm 0.2$ years, height $156.4 \pm 1.5 \mathrm{~cm}$, weight $52.5 \pm 1.6 \mathrm{~kg}$, and BMI $21.7 \pm 0.8 \mathrm{~kg} / \mathrm{m}^{2}$. Length of menstrual cycle and duration of menstrual flow of subjects during the study were $31.5 \pm 1.2$ days and $6.7 \pm$ 0.4 days, respectively. The aroma and control experiments took place on $28.7 \pm 1.3$ th day and $29.5 \pm 1.1$ th day in the late-luteal phase from the first day of menstruation, respectively. The interval between the two trials was $2.4 \pm 0.3$ days.

To confirm regular ovulatory menstrual cycles among subjects, we measured their oral temperatures and urinary ovarian hormone concentrations in the late-luteal phase $(27.9 \pm 1.0$ th day) and again in the follicular phase $(7.5 \pm 0.5$ th day), after menstruation. The basal body temperature in the late-luteal phase significantly increased from that of the follicular phase $(36.54 \pm 0.10$ vs. $36.24 \pm$ $\left.0.06^{\circ} \mathrm{C}, p=0.012\right)$. We also found significant late-luteal increase in urinary ovarian hormones compared to the follicular phase (E1: $19.2 \pm 2.7$ vs. $9.2 \pm 2.0 \mathrm{ng} / \mathrm{ml} \mathrm{Cr}, p<0.001$; PdG: $1.67 \pm 0.27$ vs. $0.28 \pm 0.05 \mu \mathrm{g} / \mathrm{ml} \mathrm{Cr,} p<0.001)$.

Autonomic nervous system activity after aroma inhalation Figure 1 represents a case of ECG R-R interval changes and the corresponding power spectra before and after inhalation of lavender aroma by a 22 -year-old subject. The HF power representing the parasympathetic nervous system activity markedly increased after the inhalation of lavender.

The baseline heart rate values before inhalation of aromas did not significantly differ between the lavender and water trials $(70.6 \pm 2.5$ vs. $69.5 \pm 2.6 \mathrm{bpm}, p=0.51)$. As shown in Figure 2, heart rate significantly decreased after the aroma stimulations (time effect: $F=12.6, p<0.001$ ), but the difference between the two trials did not reach statistical significance.

Figure 3 graphs the time course of the changes of HF power for 35 minutes after the aroma stimulations. We observed a significant increase in the HF power after the inhalation of lavender aroma in comparison with water 


\section{Lavender trial}
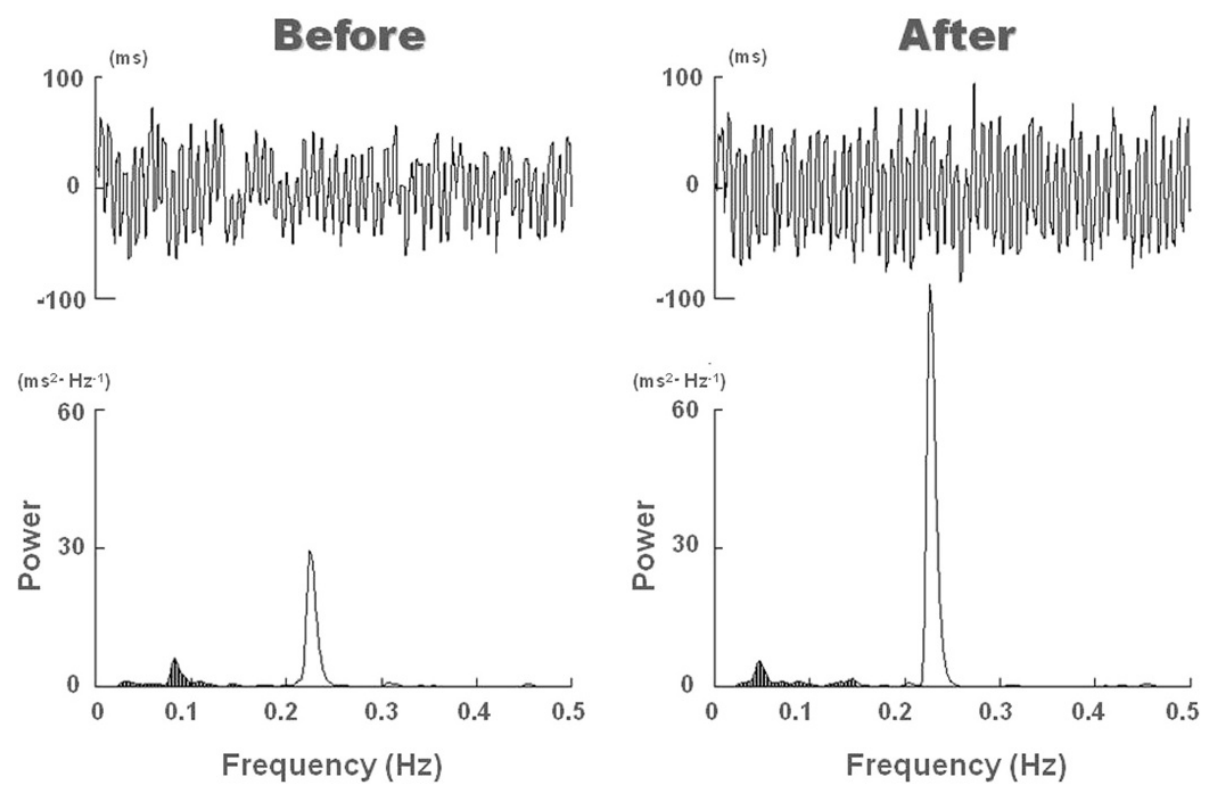

Figure 1 ECG R-R interval changes and corresponding power spectra before and after inhalation of lavender aroma by a 22-year-old subject.

(aroma effect: $F=4.50, p=0.050$; time effect: $F=5.59$, $p=0.017$; aroma $\mathrm{x}$ time effect: $F=3.17, p=0.047$ ). The rate of increase of HF power was greater at 10-15 min $(p=0.051)$ and $20-25 \mathrm{~min}(p=0.023)$ in the lavender trial compared to the control trial with water. We also calculated the ratio of LF power to HF power indicating sympathovagal balance. As suggested by Chien et al. [12], however, no statistically significant difference was detected in the relative values between the two trials.

Premenstrual emotional symptoms after aroma inhalation We found no significant difference in the baseline values of six subscales of POMS test between lavender and

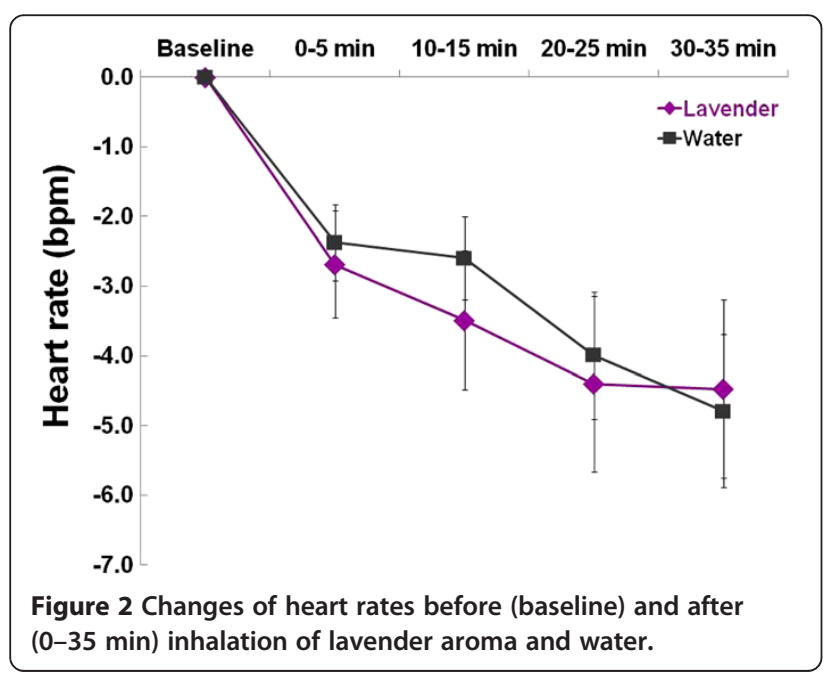

control trials. The statistical results (lavender vs. control trials) are as follows: tension-anxiety $46.4 \pm 2.5$ vs. $44.2 \pm$ $2.5, p=0.30$; depression-dejection $48.4 \pm 1.9$ vs. $46.4 \pm 1.9$, $p=0.19$; anger-hostility $52.4 \pm 3.3$ vs. $47.7 \pm 2.6, p=0.12$; vigor $44.4 \pm 2.0$ vs. $44.8 \pm 2.2, p=0.84$; fatigue $47.6 \pm 2.3$ vs. $46.4 \pm 2.7, p=0.66$; confusion $48.8 \pm 2.1$ vs. $46.7 \pm 2.1$, $p=0.18$. As Figure 4 shows, however, the subscores of depression-dejection $(p=0.045)$ and confusion $(p=$ 0.049 ) significantly decreased after the inhalation of lavender as compared to those of the control trial with water (Figure 4). Other negative symptoms-tensionanxiety, anger-hostility, and fatigue-decreased more

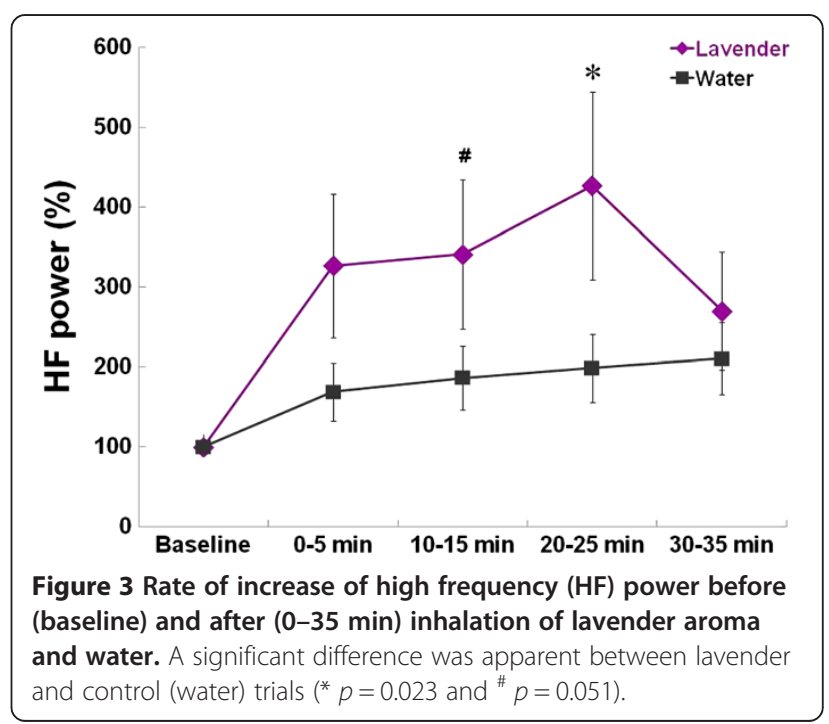




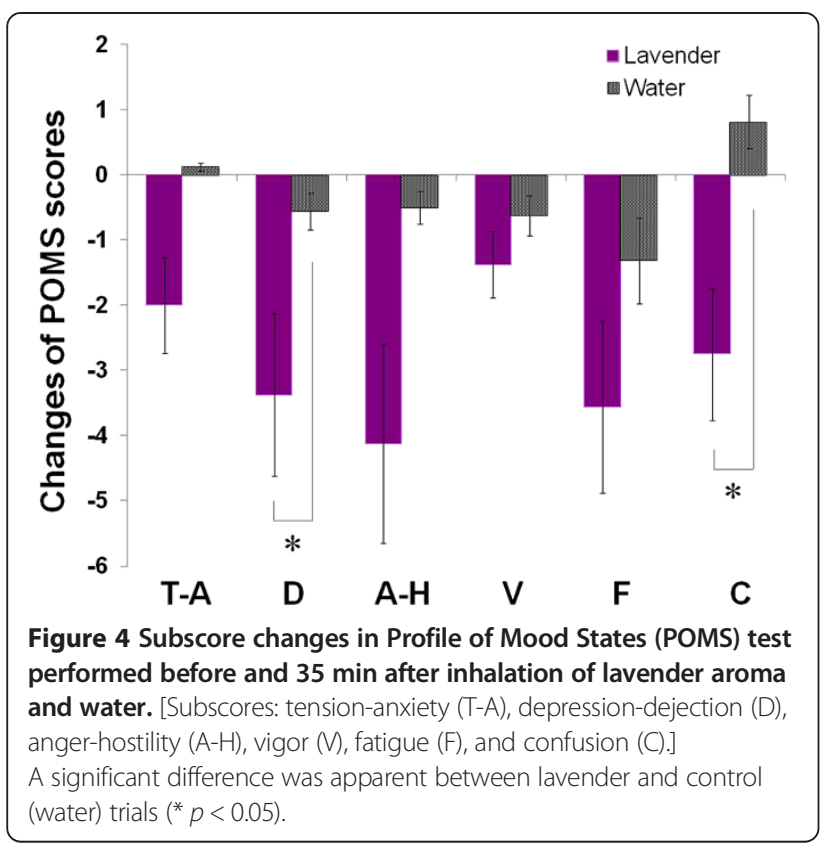

in the lavender trial, but the changes of the subscores did not statistically differ between the lavender and control trials.

\section{Discussion}

The present study investigated the effects of aromatherapy with a short-term inhalation of lavender fragrance on late-luteal autonomic nervous system activity and moderate symptom change or improvement from premenstrual emotional symptoms. Based on a literature search using the PubMed database, as of May 11, 2013, we found 756 articles on aromatherapy. However, only one article by Fukui et al. [34] addresses aromatherapy and PMS-a 20 min olfactory stimulation of saffron (Crocus sativus) significantly decreased salivary cortisol and increased $17-\beta$ estradiol, accompanying a significant decrease of anxiety scores in the late-luteal phase. To the best of our knowledge, the present study marks the first one to provide novel information on the psychophysiological effects of lavender on alleviating premenstrual emotional symptoms by using HRV power spectral analysis. The main findings reveal that HF power, reflecting parasympathetic nervous system activity, significantly increased directly after a $10 \mathrm{~min}$ inhalation of the lavender scent. This incremental effect continued at least for 25 min. In addition, the POMS test revealed that inhalation of lavender significantly decreased emotional symptoms including depression-dejection and confusion, common premenstrual symptoms, in the late-luteal phase, as long as 35 min after the aroma stimulation.

Recent research on alternative therapy has investigated both the subjective and neurophysiological effects of various plant fragrances [20]. The present study used a lavender essential oil because it has a widely applied, purportedly sedating or relaxing scent, and research has repeatedly ascribed health benefits to its aroma [6-12]. The question persists, however, how and why does lavender fragrance produce psychodynamic and physiological effects? To what extent does the autonomic nervous system, which plays a crucial role in the integrity of the mind-body connection as the functional driver of general health and wellness, contribute to efficacious outcomes produced by the fragrance? We have reviewed the literature evaluating any association of lavender and autonomic nervous system activity using HRV measurements. Saeki [35] investigated the effects of a foot-bath with and without lavender essential oil on HRV power spectra among healthy young women and demonstrated a significant increase of HF power during both types of foot-baths. The foot-bath with lavender oil, however, showed significant changes within sympathovagal balance associated with relaxation at $5 \mathrm{~min}$ after the completion of the foot-bath. According to Kuroda and colleagues [28], HF power increased more significantly at $21 \mathrm{~min}$ after a $6 \mathrm{~min}$ olfactory stimulation of (R)-(-)-linalool, one of the major scent components of lavender, compared to the control trials with water or (S)-(+)-linalool, the optical isomer of (R)-(-)-linalool. The study also found that after shortterm inhalation, the subscores of the POMS test-tension-anxiety, depression-dejection, and anger-hostilitydecreased to a greater extent than in the control trials. Chien et al. [12] investigated the effects of 12 weeks of lavender aromatherapy in midlife women (45-55 years of age) with insomnia. This study observed a significant increase in HF power after a 20 min lavender inhalation in the 4th and 12th weeks of aromatherapy and also found an apparent improvement in sleep quality after the intervention. Despite the differences in experimental designs and conditions, these earlier investigations support our findings indicating that short-term lavender inhalation modulates HRV with the predominance of parasympathetic nervous system activity while inducing soothing effects.

While we mentioned the paucity of information on the effects of lavender inhalation and PMS, one study by Shimizu [29] published in a Japanese medical journal reported on the negative subscales of the POMS testtension-anxiety, depression-dejection, anger-hostility and confusion-significantly decreasing and a positive subscore, vigor, increasing in the luteal phase after therapeutic intervention with a $15 \mathrm{~min}$ inhalation of linalyl acetate, another major component of lavender, twice a day (morning and evening) daily for one menstrual cycle. This study did not measure any physiological data including HRV measurements. Considering three previous investigations mentioned above [12,28,35] and the present research together with the findings by Shimizu [29], however, lavender could serve as a potential anti-PMS fragrance, especially for 
alleviating negative emotional stress appearing premenstrually. Although the detailed mechanism of the efficacy of lavender on premenstrual symptoms remains unknown, the significant increase in HF power after the inhalation of lavender found in the present study further suggests that lavender interacts with parasympathetic nervous system activity to modulate the cluster of premenstrual psychoemotional symptoms.

From a pharmacological point of view, Herz [20] showed that lavender acts postsynaptically and suggested that lavender modulates the activity of cyclic adenosine monophosphate (cAMP). A reduction in cAMP activity is associated with sedation. Linalool, a principal component of lavender, has also been found to inhibit glutamate binding, which may have sedative effects. To further explore the psychoneurophysiological mechanism of aromatherapy with lavender, neuroimaging techniques provide new insights into the role of the brain in correlation with autonomic modulation. Olfaction is mediated by chemoreceptors of olfactory cells located in the nasal mucosae and olfactory neurons in the olfactory bulb. Olfactory information is further projected to the primary olfactory regions in the brain and most of these brain regions are strongly connected to or are part of the limbic system, the center of autonomic function and emotion [6]. By applying a combination of HRV power spectral analysis and positron emission tomography (PET) examination, Duan and colleagues [36] found a significant increase in HF power, indicating parasympathetic nervous system acidity after a lavender aroma treatment, as we demonstrated in the present study. Simultaneous PET measurements, detecting the specific regional metabolic activations and reductions after the treatment, suggested that the lavender fragrance induced not only physical relaxation but also improved mental function.

The present study used two kinds of aroma, lavender and water as a control. To avoid placebo effects, we did not inform subjects of which fragrance we would use for the experiment or that one of the two trials could be a control trial, during the entire study period. We cannot, however, completely deny the possibility that the participants would have noticed the difference when they inhaled the aroma of water. A study using lavender together with other plant fragrances possessing different psychological and pharmacological effects could extend the present research by scrutinizing the effects of aromatherapy on premenstrual symptoms while protecting against a placebo effect.

While this study produced intriguing findings-augmentation of parasympathetic nerve activity accompanying sedative effects on negative premenstrual emotional status by lavender stimulation-it employed a relatively small sample size. In addition, the intensity of premenstrual symptoms among subjects ranged from mild to moderate and represents the premenstrual molimina experienced by a greater percentage of reproductive age women, compared to PMS or PMDD [1-3]. Our previous research has revealed that the menstrual cyclic patterns of autonomic nervous system activity significantly differ among women with premenstrual molimina, PMS and PMDD [21-24]. We thus need to conduct future studies with a larger sampling of women with different degrees of symptoms-from premenstrual molimina to PMDD together with premenstrual exacerbation [26] to further explore the therapeutic efficacy of plant fragrances including lavender on premenstrual symptomatology. Premenstrual discomfort remains enigmatic, but researchers continue to unveil its etiology, which includes estrogen excess, progesterone deficiency, decreases in serotonergic tone, alteration of central GABA function, and opioids withdrawal $[1-3,26]$. These theoretical models lead us to speculate that the sophisticated independent menstrual-cyclic fluctuation of ovarian hormones, estrogen and progesterone, could ingeniously interact with the central nervous, autonomic nervous, endocrine and immune systems. Taken together, interdisciplinary research is also required to scrutinize the psychological, neurophysiological, and pharmacological functions of lavender essential oils for alleviating the undefined symptom complex appearing so commonly premenstrually.

In conclusion, the present study, using HRV power spectral analysis, investigated the efficacy of lavender aromatherapy as a potential therapeutic modality of premenstrual symptoms from the perspective of autonomic nervous system activity. This study indicates that shortterm inhalation of lavender could alleviate premenstrual emotional symptoms and could, at least in part, contribute to the improvement of parasympathetic nervous system activity. The present study further implies that HRV can be used to evaluate the efficacy of aromatherapy (using various kinds of fragrances) in relieving premenstrual symptoms, and ultimately, promote the mind and body health of women.

\section{Competing interests}

The authors declare that they have no competing interest.

\section{Authors' contributions}

TM conceptualized and designed the study, collected and analyzed the data, performed the statistical analysis, interpreted the results, and drafted the manuscript. HA participated in the design and coordination of the present study and provided clinical evidence of premenstrual symptoms from his gynecological research as well as practical suggestions to interpret the results. TH contributed to analyzing data, helped to interpret the results with productive and valuable comments, and provided neuroendocrinological information to develop the present research. All authors read and approved the final manuscript.

\section{Acknowledgements}

The authors wish to express their appreciation to all volunteers for their dedicated participation in this study. The Japan Society for the Promotion of Science, Grant-in-Aid for Scientific Research (C) 21500669, supported this research. 


\section{Author details}

${ }^{1}$ Department of Health Education, Faculty of Education, Shitennoji University, 3-2-1 Gakuenmae, Habikino Osaka 583-8501, Japan. '2Ohgimachi Ladies Clinic, Center for Advanced Reproductive Endocrinology and Infertility, Osaka, Japan. ${ }^{3}$ Graduate School of Human and Environmental Studies, Kyoto University, Kyoto, Japan.

Received: 12 March 2013 Accepted: 28 May 2013

Published: 31 May 2013

\section{References}

1. Rapkin AJ, Winer SA: Premenstrual syndrome and premenstrual dysphoric disorder: quality of life and burden of illness. Expert Rev Pharmacoecon Outcomes Res 2009, 9:157-170.

2. O'Brien PM, Bäckström T, Brown C, Dennerstein L, Endicott J, Epperson CN, Eriksson E, Freeman E, Halbreich U, Ismail KM, Panay N, Pearlstein T, Rapkin A, Reid R, Schmidt P, Steiner M, Studd J, Yonkers K: Towards a consensus on diagnostic criteria, measurement and trial design of the premenstrual disorders: the ISPMD Montreal consensus. Arch Womens Ment Health 2011, 14:13-21.

3. Matsumoto $\mathrm{T}$, Asakura $\mathrm{H}$, Hayashi T: Biopsychosocial aspects of premenstrual syndrome and premenstrual dysphoric disorder. Gynecol Endocrinol 2013, 29:67-73.

4. Dante G, Facchinetti F: Herbal treatments for alleviating premenstrual symptoms: a systematic review. J Psychosom Obstet Gynaecol 2011, 32:42-51.

5. Tillett J, Ames D: The uses of aromatherapy in women's health. J Perinat Neonatal Nurs 2010, 24:238-245.

6. Price S, Price L: Aromatherapy for health professionals. 4th edition. London: Churchill Livingstone Elsevier; 2012

7. Hadi N, Hanid AA: Lavender essence for post-cesarean pain. Pak J Biol SC 2011, 14:664-667.

8. Conrad P, Adams C: The effects of clinical aromatherapy for anxiety and depression in the high risk postpartum woman - a pilot study. Complement Ther Clin Pract 2012, 18:164-168.

9. Apay SE, Arslan S, Akpinar RB, Celebioglu A: Effect of aromatherapy massage on dysmenorrhea in Turkish students. Pain Manag Nurs 2012, 13:236-240.

10. Hur MH, Yang YS, Lee MS: Aromatherapy massage affects menopausal symptoms in Korean climacteric women: a pilot-controlled clinical trial. Evid Based Complement Alternat Med 2008, 5:325-328.

11. Igarashi T: Physical and psychologic effects of aromatherapy inhalation on pregnant women: a randomized controlled trial. J Altern Complement Med 2013. Feb 14 [Epub ahead of print]

12. Chien LW, Cheng SL, Liu CF: The effect of lavender aromatherapy on autonomic nervous system in midlife women with insomnia. Evid Based Complement Alternat Med 2012, Article ID 740813:8.

13. Chandola T, Heraclides A, Kumari M: Psychophysiological biomarkers of workplace stressors. Neurosci Biobehav Rev 2010, 35:51-57.

14. Dishman RK, Nakamura Y, Garcia ME, Thompson RW, Dunn AL, Blair SN: Heart rate variability, trait anxiety, and perceived stress among physically fit men and women. Int J Psychophysiol 2000, 37:121-133.

15. Hughes JW, Stoney CM: Depressed mood is related to high-frequency heart rate variability during stressors. Psychosom Med 2000, 62:796-803.

16. Gorman JM, Sloan RP: Heart rate variability in depressive and anxiety disorders. Am Heart J 2000, 140:S77-S83.

17. Cohen H, Kotler M, Matar MA, Kaplan Z, Loewenthal U, Miodownik H, Cassuto $Y$ : Analysis of heart rate variability in posttraumatic stress disorder patients in response to a trauma-related reminder. Biol Psychiatr 1998, 44:1054-1059.

18. Freeman $\mathrm{R}$, Komaroff $\mathrm{AL}$ : Does the chronic fatigue syndrome involve the autonomic nervous system? Am J Med 1997, 102:357-364.

19. Thayer JF, Ahs F, Fredrikson M, Sollers JJ 3rd, Wager TD: A meta-analysis of heart rate variability and neuroimaging studies: implications for heart rate variability as a marker of stress and health. Neurosci Biobehav Rev 2012, 36:747-756.

20. Herz RS: Aromatherapy facts and fictions: a scientific analysis of olfactory effects on mood, physiology and behavior. Int J Neurosci 2009, 119:263-290.

21. Matsumoto T, Ushiroyama T, Morimura M, Moritani T, Hayashi T, Suzuki T, Tatsumi N: Autonomic nervous system activity in the late luteal phase of eumenorrheic women with premenstrual symptomatology. J Psychosom Obstet Gynaecol 2006, 27:131-139.
22. Matsumoto T, Ushiroyama T, Tatsumi N: Lower peripheral circulation in eumenorrheic young women with premenstrual symptoms. Biopsychosoc Med 2007, 1:8

23. Matsumoto T, Ushiroyama T, Kimura T, Hayashi T, Moritani T: Altered autonomic nervous system activity as a potential etiological factor of premenstrual syndrome and premenstrual dysphoric disorder. Biopsychosoc Med 2007, 1:24.

24. Matsumoto T, Asakura $\mathrm{H}$, Hayashi T: Increased salivary chromogranin A in women with severe negative mood states in the premenstrual phase. J Psychosom Obstet Gynaecol 2012, 33:120-128.

25. Moos $\mathrm{RH}$ : The development of a menstrual distress questionnaire. Psychosom Med 1968, 30:853-867.

26. Kessel B: Premenstrual syndrome. Advances in diagnosis and treatment Obstet Gynecol Clin North Am 2000, 27:625-639.

27. Kiecolt-Glaser JK, Graham JE, Malarkey WB, Porter K, Lemeshow S, Glaser R: Olfactory influences on mood and autonomic, endocrine, and immune function. Psychoneuroendocrinology 2008, 33:328-339.

28. Kuroda K, Inoue N, Ito Y, Kubota K, Sugimoto A, Kakuda T, Fushiki T: Sedative effects of the jasmine tea odor and (R)-(-)-linalool, one of its major odor components, on autonomic nerve activity and mood states. Eur J Appl Physiol 2005, 95:107-114.

29. Shimizu K: Effect of aromatherapy on premenstrual syndrome-Verification of linalyl acetate. Ochanomizu lgaku Zasshi 2008, 56:15-25 (in Japanese)

30. Hayashi T, Masuda I, Shinohara M, Moritani T, Nakao K: Autonomic nerve activity during physical exercise and postural change: investigations by power spectral analysis of heart rate variability. Jpn J Biochem Exerc 1994, 6:30-37 (in Japanese).

31. Moritani T, Kimura T, Hamada T, Nagai N: Electrophysiology and kinesiology for health and disease. J Electromyogr Kinesiol 2005, 15:240-255.

32. Kimura T, Matsumoto T, Akiyoshi M, Owa Y, Miyasaka N, Aso T, Moritani T: Body fat and blood lipids in postmenopausal women are related to resting autonomic nervous system activity. Eur J Appl Physiol 2006, 97:542-547.

33. Yokoyama K, Araki S: The Japanese version of the POMS manual. Kaneko Shobo: Tokyo; 1994

34. Fukui $H$, Toyoshima K, Komaki R: Psychological and neuroendocrinological effects of odor of saffron (Crocus sativus). Phytomedicine 2011, 18:726-730.

35. Saeki Y: The effect of foot-bath with or without the essential oil of lavender on the autonomic nervous system: a randomized trial. Complement Ther Med 2000, 8:2-7.

36. Duan X, Tashiro M, Wu D, Yambe T, Wang Q, Sasaki T, Kumagai K, Luo Y, Nitta S, Itoh M: Autonomic nervous function and localization of cerebral activity during lavender aromatic immersion. Technol Health Care 2007, 15:69-78.

doi:10.1186/1751-0759-7-12

Cite this article as: Matsumoto et al:: Does lavender aromatherapy alleviate premenstrual emotional symptoms?:

a randomized crossover trial. BioPsychoSocial Medicine 2013 7:12

\section{Submit your next manuscript to BioMed Central and take full advantage of:}

- Convenient online submission

- Thorough peer review

- No space constraints or color figure charges

- Immediate publication on acceptance

- Inclusion in PubMed, CAS, Scopus and Google Scholar

- Research which is freely available for redistribution 